\title{
Two nonlinear optical processes in Z-scan curves of a bleached photographic film
}

\author{
E. Reynoso-Lara 1*, A. Balbuena-Ortega², Y. E. Bravo-García', J. A. Dávila-Pintle', M. L. Arroyo-Carrasco \\ and M. D. Iturbe-Castillo ${ }^{3}$
}

\begin{abstract}
Background: In this work, the experimental behavior observed in the z-scan curves of a sample of bleached photographic film for different incident powers is theoretically modeled.

Methods: Two models are modified in order to fit the experimental z-scan curves. The Focal Length Dependent on a power of the beam radius (FLD) model and the Nonlocal (NL) model.

Results: As a result of the fitting, it is not possible to fit the experimental results considering only one contribution during the z-scan experiment.

Conclusion: The results demonstrated that two contributions were needed to reproduce completely the experimental z-scan curves in both models.
\end{abstract}

Keywords: Nonlinear optics, Z-scan, Photographic film

\section{Background}

A photographic film is a photosensitive material that when is exposed to light undergoes physical changes. The film is composed of silver halide crystals (silver bromide generally) that absorbs the optical radiation to form a latent image. After chemical processing (develop), the latent image turns into a visible negative image (as R. Wayne explained detailed in [1]). Nowadays, photographic films are still used in many areas, mainly as a phase modulator or to record gratings or phase holograms [2-9]. A bleached photographic film is a developed photographic film where all the opaque regions are transformed into transparent by another chemical processing. In [10] it was reported that bleached photographic film can exhibit nonlinear optical behavior when is illuminated with $\mathrm{cw}$ laser at powers of some milliwatts.

The z-scan technique is a widely used method to obtain the nonlinear refractive index of different materials. Since it was proposed in $[11,12]$, many materials of several types have been characterized with this technique. Different

\footnotetext{
* Correspondence: edmundo.reynoso@correo.buap.mx

${ }^{1}$ Benemérita Universidad Autónoma de Puebla, Av. San Claudio y 18 sur,

72570 Puebla, Puebla, Mexico

Full list of author information is available at the end of the article
}

theoretical approaches have been also suggested to describe the z-scan curves mainly for materials with a Kerr nonlinear response; the most complete of these theories was published in [13].

In this paper, taking into account the experimental characterization reported in [10], we proposed that in some materials the nonlinear response can change for each position: something in the medium is modify that affects to the beam in a different way than in the previous position. We propose that this response can be modeled as one parameter that changes from one value to other in a smooth way. We include this idea in two models to describe the experimental behavior observed in [10]. One of the models is based on the idea of that a lens is photoinduced in the samples with a focal length that depends on the incident beam radius to some power [14] but this power changes with the position of the sample. The second model is based on the nonlocality of the nonlinear response of the sample [15], that also can change with the position. In the next section we describe and reproduce the experimental results reported in [10]. Then, a brief description of the models is presented. Both models are used to fit one set of the experimental results, first considering one and then two contributions. Finally the conclusions of the work are presented. 


\section{Antecedents}

The z-scan experimental characterization reported in [10] was made at the wavelengths 457, 476, 488, 496 and $514 \mathrm{~nm}$ from an Argon laser and at $633 \mathrm{~nm}$ from a HeNe laser. A beam waist of $15 \mu \mathrm{m}$ was reported for the experiments with the Argon laser beam and of $30 \mu \mathrm{m}$ for the $\mathrm{HeNe}$ laser beam. The on axis intensity detection was made with a photodetector located at approximately $80 \mathrm{~cm}$ from the waist. First z-scan measurements were made for a power of $1 \mathrm{~mW}$, then the power was increased to $5 \mathrm{~mW}$ and finally to $10 \mathrm{~mW}$. Immediately, new z-scan curves were obtained reducing the power to $5 \mathrm{~mW}$ and after to $1 m W$, keeping the rest of the experimental conditions without change. The results obtained are reproduced in Fig. 1. The main conclusions of that paper were that the bleached photographic film behaves as a negative nonlinear media with a nonlinearity of the thermal type $[16,17]$, after comparison of the experimental results with numerical simulations. It is also mentioned that intensities higher than $200 \mathrm{~mW} / \mathrm{cm}^{2}$, creates permanent changes in the film.

\section{Method \\ Fitting models \\ Background}

The theoretical models, which we are going to use to fit the experimental z-scan curves, consider that the incident beam to the sample in the z-scan experiment is of Gaussian type, i.e. an electric field of the form:

$$
E(r, z)=A_{0} \frac{W_{0}}{W(z)} \exp \left[-\frac{r^{2}}{W(z)^{2}}\right] \exp \left[-i k z-i k \frac{r^{2}}{2 R(z)}+i \varsigma(z)\right]
$$

where,

$$
\begin{aligned}
& W(z)=W_{0}\left[1+\left(z / z_{0}\right)^{2}\right]^{1 / 2}, \\
& R(z)=z\left[1+\left(z_{0} / z\right)^{2}\right], \\
& \varsigma(z)=\tan ^{-1}\left(z / z_{0}\right)
\end{aligned}
$$

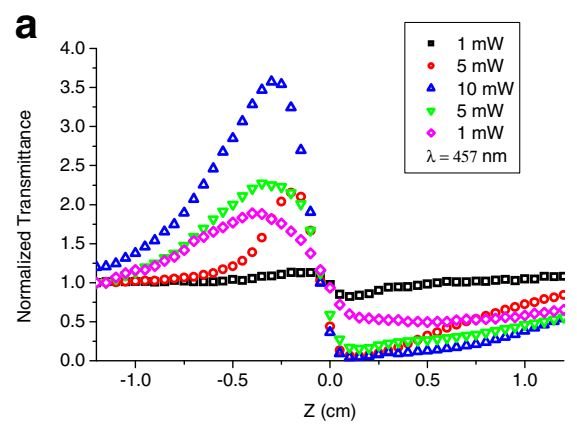

C

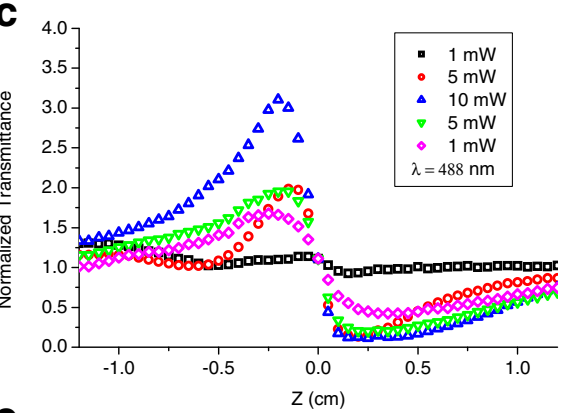

e

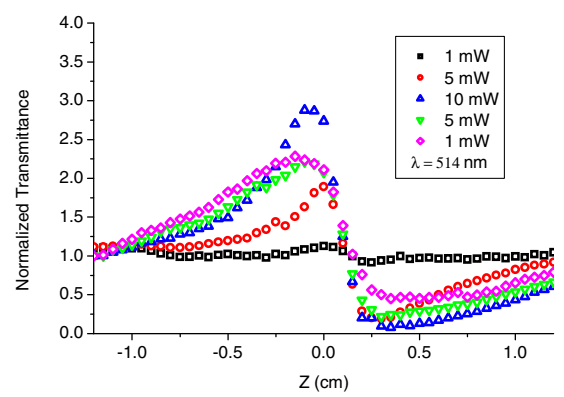

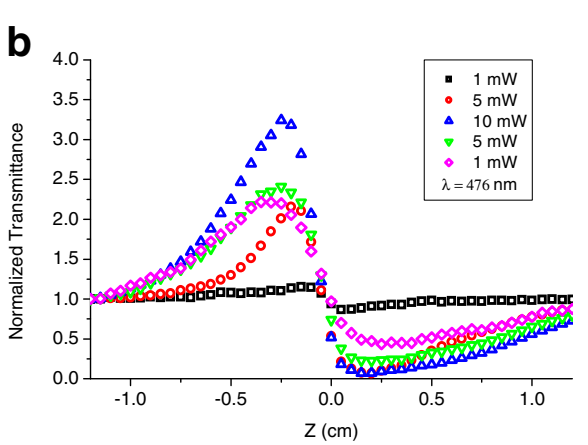

d

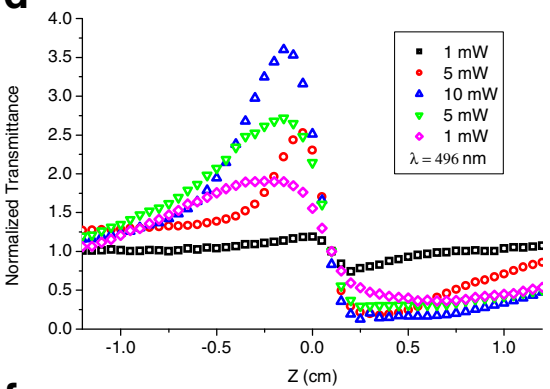

f

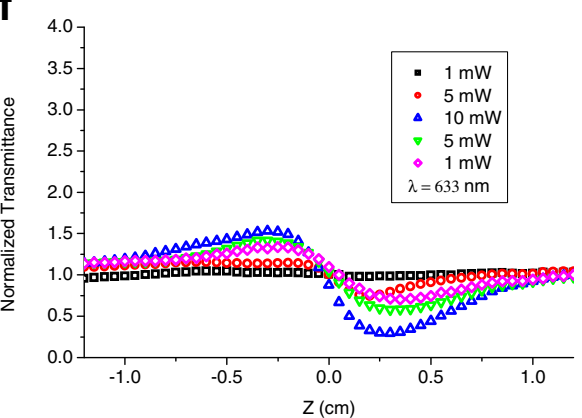

Fig. 1 Experimental z-scan curves of the photographic film to the wavelengths of: (a) 457, (b) 476, (c) 488, (d) 496, (e) 514 and (f) 633 nm. All of them to the incident powers of: 1, 5, 10, 5 and $1 \mathrm{~mW}$ 
here, $A_{O}$ is a constant and $W_{O}$ is the waist beam. $W(z)$ and $R(z)$ are the beam radius and wavefront radius of curvature, respectively. $z_{0}=\pi \mathrm{W}_{0}^{2} / \lambda$ is the Rayleigh length, $k=2 \pi / \lambda$ wavenumber and $\lambda$ is the wavelength of the beam.

The width of the sample going to be considered less than $z_{0}$, the thin sample approximation is fulfilled. And the detection in the z-scan experiment is made at a distance larger than $z_{0}$, far field approximation is fulfilled.

\section{Focal length dependent on a power of the beam radius (FLD) model}

This model is based on the idea that when a thin nonlinear medium is illuminated by a Gaussian beam, the effect over the beam can be described as that of a lens with a focal length $F$ given by [14]:

$$
F=a_{s} W^{s}(z)
$$

where $a_{s}$ is a constant and $s$ is real number larger than 1 that is associated to the type of nonlinearity exhibited by the medium. In general, the on axis normalized intensity transmittance, $T$, in a $\mathrm{z}$-scan experiment can be calculated from:

$$
T=\frac{F^{2}}{z_{0}^{2}+(F-z)^{2}} .
$$

\section{Nonlocal (NL) model}

This model considers that when a Gaussian beam, of amplitude $E(r, z)$, illuminates a thin sample with solely a refractive nonlinearity, the output field, $E_{\text {out }}$, after the thin medium, is given by

$$
E_{\text {out }}=E(r, z) \exp [-i \Delta \phi(r)]
$$

where the nonlinear phase change $\Delta \phi(r)$ is given by:

$$
\Delta \phi(r)=\frac{\Delta \Phi_{0}}{\left(1+\left(z / z_{0}\right)^{2}\right)^{m / 2}} \exp \left(-m r^{2} / W(z)^{2}\right)
$$

where $\Delta \Phi_{0}$ is the maximum nonlinear phase shift at $z=0$, and $m$ is a real positive number that characterize the spatial nonlocality of the nonlinear response. The far field intensity can be calculated numerically taking the Fourier transform of Eq. 4 for a given value of $\Delta \Phi_{0}$ and $m$.

It is possible, using the electric field described in Eq. 4, to obtain analytical formulas for the normalized transmittance through Gaussian Decomposition method [18]. The formula proposed is:

$$
T_{m}\left(z, \Delta \Phi_{0}\right)=\left|\sum_{n=0}^{N} \frac{1}{n !}\left[\frac{\Delta \Phi_{0}(z)}{\left(1+x^{2}\right)^{m / 2}}\right]^{n} \frac{i^{n}(x+i)}{x+i(m n+1)}\right|^{2},
$$

where $x=z / z_{0}$ and $N$ is an integer number and again $\Delta \Phi_{0}$ is the maximum on-axis photoinduced phase shift of the wavefront of the beam.

\section{Results}

Fittings

In this section we are going to present, as an example, the fitting of the experimental results reported in [10] at a wavelength of $457 \mathrm{~nm}$ for incident power of: 1, 5 and $10 \mathrm{~mW}$. The z-scan curves when the power, after reaching the $10 \mathrm{~mW}$, was reduced are not considered in this work. Fitting with only one contribution is first presented in order to justify the necessity of considering more than one nonlinear response in the z-scan curve.

\section{Fitting with only one contribution}

Considering the formula (3) for the FLD model with specific value of parameters $s$ and $a_{s}$ (where this parameter mostly change with the incident power), it was not possible to obtain a good correspondence between numerical and experimental results. However, we noticed that some parts of numerical curve reproduced with good correspondence the experimental results. The same behaviour was observed with the NL model. It is important to mention that the curve for $1 m W$ z-scan is reproduced all with only one contribution $\left(s=4, a_{s}=-10 \times 10^{10}\left[\mathrm{~m}^{-3}\right]\right)$, see Figs. 2 and 3 . In Fig. 2 the peaks were reproduced with good agreement with the following values for the $5 \mathrm{~mW}$ curve: $s=4, a_{s}=-10 \times 10^{9}\left[\mathrm{~m}^{-3}\right]$; and for the $10 \mathrm{~mW}$ curve: $\mathrm{s}=2, a_{s}=-48 \times 10^{3}\left[\mathrm{~m}^{-1}\right]$. In Fig. 3 , the valleys were

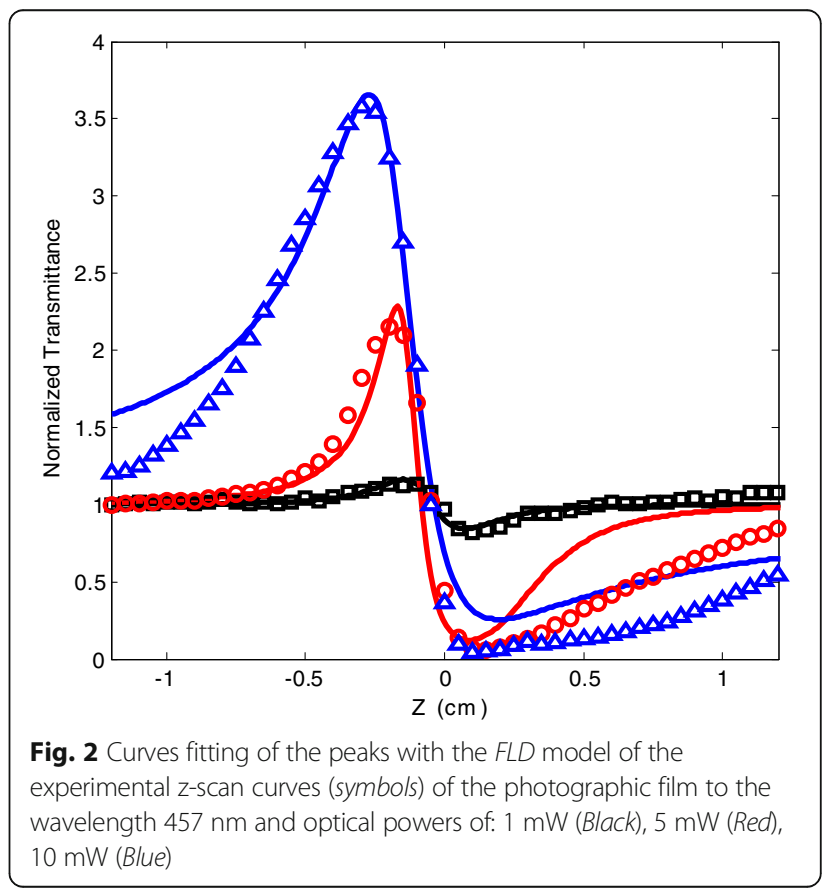




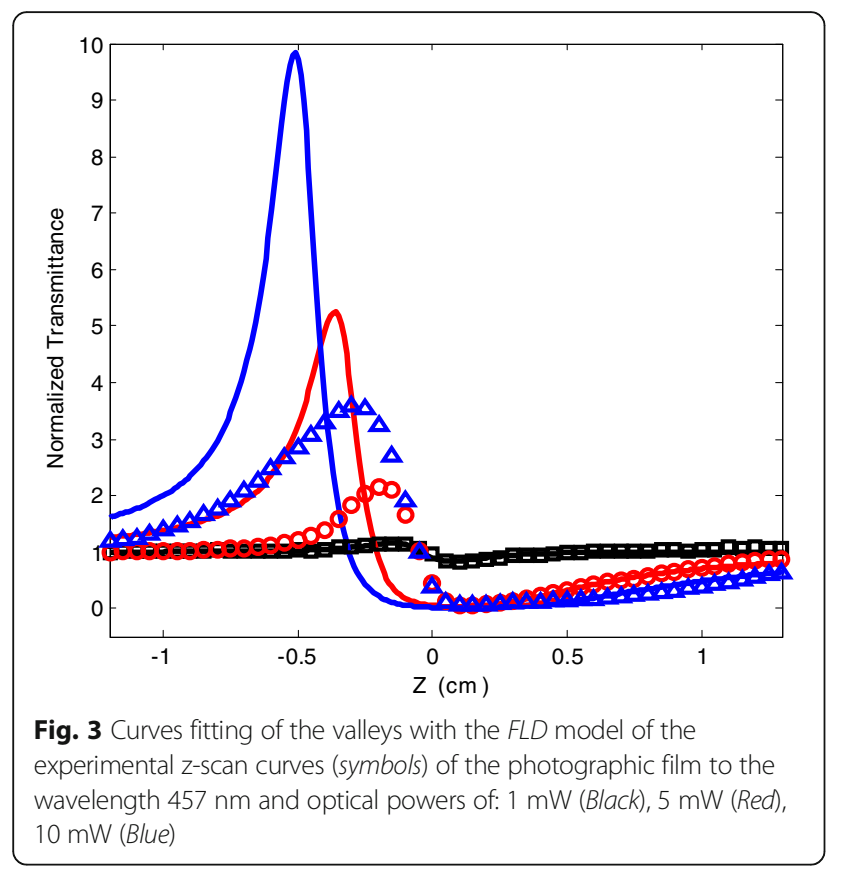

reproduced with the following values for the $5 \mathrm{~mW}$ curve: $\mathrm{s}=3, a_{s}=-8.4 \times 10^{6}\left[\mathrm{~m}^{-2}\right]$; and for the $10 \mathrm{~mW}$ curve: $s=3, a_{s}=-4 \times 10^{6}\left[\mathrm{~m}^{-2}\right]$.

In the same way, with the nonlocal model the curve for $1 m W \mathrm{z}$-scan is reproduced all with only one contribution $\left(m=2.5, \Delta \Phi_{0}=-0.3 \pi\right)$ and the best fit for the peaks was obtained with the following values for the $5 m W$ curve where $m=2.8$ and $\Delta \Phi_{0}=-6 \pi$; for the $10 m W$ curve $m=0.4$ and $\Delta \Phi_{0}=-2.3 \pi$, see Fig. 4 . Then, the valleys were fitted obtaining that the $5 \mathrm{~mW}$ curve where $m=0.4$ and $\Delta \Phi_{0}=-3 \pi$; for the $10 m W$ curve $m=0.5$ and $\Delta \Phi_{0}=-6 \pi$, see Fig. 5 .

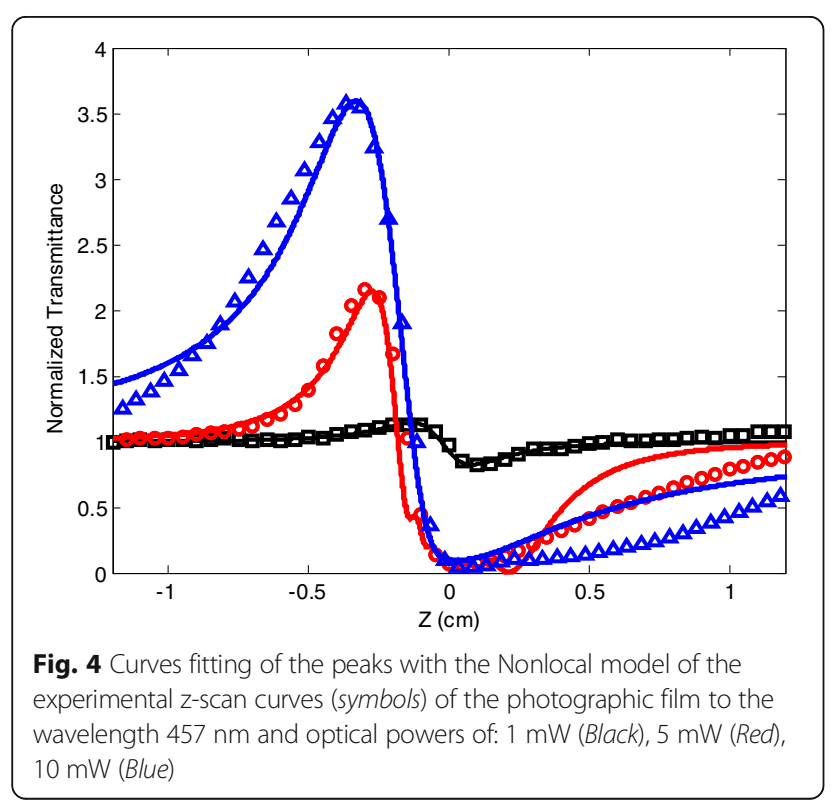

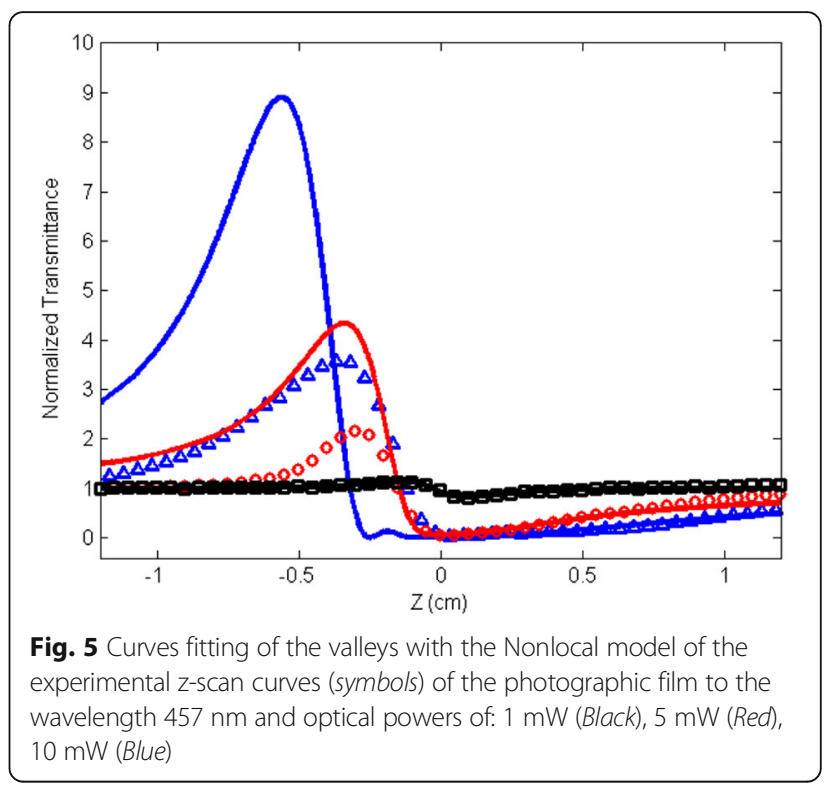

The previous analysis demonstrate that the experimental z-scan curves cannot be fitted completely with only one pair of values for the FLD or NL models, in particular the curves belonging to the optical powers of 5 and $10 \mathrm{~mW}$. It is clear that the material, as it was proposed in [10], is affected by the light intensities larger than $200 \mathrm{~mW} / \mathrm{cm}^{2}$, creating a change in the film that modifies how the light is transmitted by the sample. In order reproduce the behaviour obtained by the experimental z-scan curves we propose considers more than one contribution, during the $\mathrm{z}$-scan, in the FLD and NL models.

\section{Fitting with two nonlinear contributions}

The maximum intensity of a Gaussian beam is reached at the waist for this reason we are going to consider that in the photographic film one response must be take into account for negative $z$ and other for positive $z$. This means that to obtain the best fit of the experimental z-scan curves are needed two nonlinear responses. Consequently, it is necessary to consider two pairs of parameter values $\left(s, a_{s}\right)$ or $\left(m, \Delta \Phi_{0}\right)$, depending of the theoretical model; that is, one pair for the peak and another for the valley of the curve. To achieve this sudden change, we make use of the Heaviside step function, in order to have one response for negative $z$ and another for positive $z$. In fact, observing the Figs. 2, 3 and 4, 5 indicate that change between two nonlinear optical processes is in a very small region which is in the negative slope of the transition peak-valley. We consider an approximation of Heaviside Step function of the form:

$$
H \operatorname{Step}(z)=-0.5[\tanh (l z) \pm 1]=H S
$$

where $l$ is the rate of change between the maxima and 
minimum of the function. This $l$ parameter has to be matched with the slope of the curve in the region close to $z=0$. Then in general the transmittance can be obtained, in a first approximation, as the sum of that obtained with one set of values for negative $z\left(T^{p k}\right)$ with that obtained for positive $z\left(T^{v y}\right)$.

The Eqs. (8) and (9) are two equations that help to exactly fit the complete curves with two nonlinear optical contributions in both FLD and $N L$ models, respectively.

$$
\begin{aligned}
& T_{F L D}(z)=H S_{p} T_{a_{s}}^{P k}+H S_{v} T_{a_{s}}^{v y}, \\
& T_{N L}(z)=H S_{p} T_{m}^{P k}+H S_{v} T_{m}^{v y} .
\end{aligned}
$$

The superscripts $p k$ and $v y$ are for peak and valley as well as $H S_{p}$ and $H S_{v}$ functions are the Heaviside Step functions to weight the peaks and valleys respectively in each model.

Figures 6 and 7 show the exact curve fitting with the FLD and nonlocal models.

The curves were calculated using the values reported in the Table 1 for the two models and different incident powers. For the FLD model the values of $s$ were integers, a value of 4 is associated to a Kerr mechanism of nonlinearity and 2 is associated to a thermal, a value of 3 has been associated to a reorientational mechanism [19]. For NL model values of $m$ larger than 2 are associated with nonlinear phase changes that spatially are extended to region narrower than the incident intensity and values smaller than 2 extend in a broader region [15].

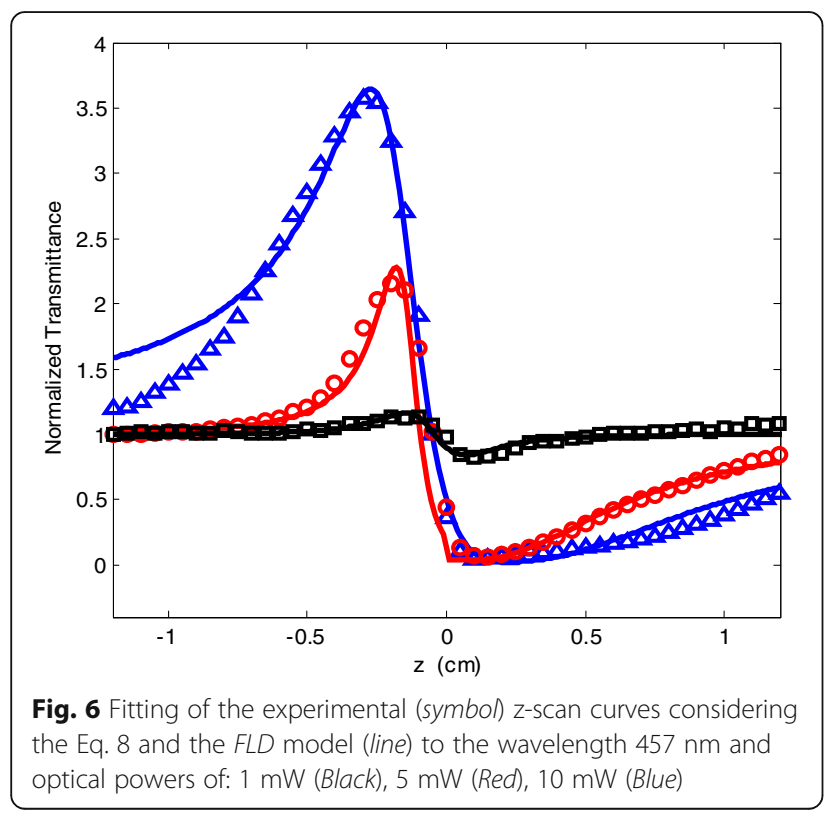

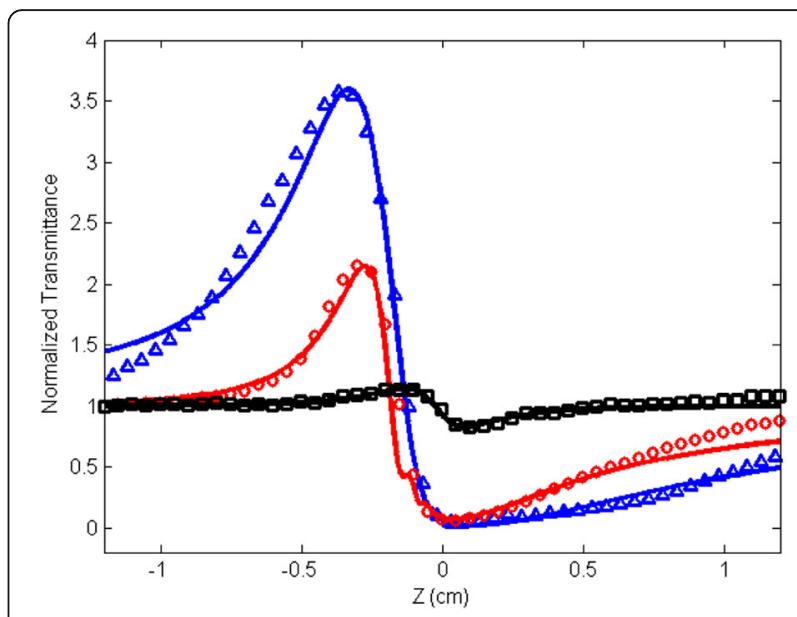

Fig. 7 Fitting of the experimental (symbol) z-scan curves considering the Eq. 9 and the nonlocal model (line) to the wavelength $457 \mathrm{~nm}$ and optical powers of: $1 \mathrm{~mW}$ (Black), $5 \mathrm{~mW}$ (Red), $10 \mathrm{~mW}$ (Blue)

\section{Discussion}

Photographic film is an emulsion of gelatin made of millions of light sensitive silver halide crystals distributed uniform over a substrate (glass or plastic). When the light strikes these crystals a reaction (important change) takes place. The silver halides of the film that were struck by light have been rearranged changing the density $(D)$ as a function of the light exposure $(E)[1] . D=\varepsilon c d$, according to Lambert-Beer Law, where the extinction coefficient $\varepsilon\left[\mathrm{m}^{2} / \mathrm{mol}\right]$ characterizes the ability to the silver grains to interact with light, $c$ is the concentration of silver grains $\left[\mathrm{mol} / \mathrm{m}^{3}\right]$, and $d$ is the thickness of the film $[\mathrm{m}]$. Exposure is equal to product of incident light intensity [photons $\left./ \mathrm{s} \mathrm{m}^{2}\right]$ and time $[\mathrm{s}]$. Therefore, with exposure extremely short the influence of light is low; however, at high light levels or long exposure times, the chance of a grain capturing light is so high such that the sample changed its physical properties permanently due to high intensity that was exposed (around the focal plane); this is the reason because the experimental $\mathrm{z}$-scan curves obtained by second time with the powers of 5 and $10 \mathrm{~mW}$, were not equals as the first measurements. Also, it is important to note that the nonlinear response is higher at blue end region $(457 \mathrm{~nm})$ than in red region $(633 \mathrm{~nm})$, it is result to the ability to absorb stronger in that region, as can be observed in Figure 1 of [10]. The fitting to the experimental results demonstrates that the nonlinear refractive index of the photographic film changes as the intensity of the incident beam overpowers certain value. In this work it was assumed that at the position of the waist, of the beam used for the z-scan characterization, the sample change of nonlinearity (FDL model) or nonlocality (NL model). Besides, the optical nonlinear response is highly nonlocal with higher levels of 
Table 1 Parameters values to exact fit the experimental z-scan curves of the photographic film with the FLD and NL models

\begin{tabular}{|c|c|c|c|c|c|c|c|c|}
\hline \multirow{3}{*}{$\begin{array}{l}\text { Optical } \\
\text { Power } \\
(\mathrm{mW})\end{array}$} & \multicolumn{4}{|c|}{ FLD model } & \multicolumn{4}{|c|}{$\mathrm{NL}$ model } \\
\hline & \multicolumn{2}{|c|}{ Peaks } & \multicolumn{2}{|c|}{ Valleys } & \multicolumn{2}{|c|}{ Peaks } & \multicolumn{2}{|c|}{ Valleys } \\
\hline & s & $a_{5}$ & s & $a_{\mathrm{s}}$ & $m$ & $\Delta \Phi_{0}$ & $m$ & $\Delta \Phi_{0}$ \\
\hline 1 & 4 & $-10 \times 10^{10}\left[\mathrm{~m}^{-3}\right]$ & 4 & $-10 \times 10^{10}\left[\mathrm{~m}^{-3}\right]$ & 2.5 & $-0.3 \pi$ & 2.5 & $-0.3 \pi$ \\
\hline 5 & 4 & $-10 \times 10^{9}\left[\mathrm{~m}^{-3}\right]$ & 3 & $-8.4 \times 10^{6}\left[\mathrm{~m}^{-2}\right]$ & 2.8 & $-6 \pi$ & 0.4 & $-3 \pi$ \\
\hline 10 & 2 & $-48 \times 10^{3}\left[\mathrm{~m}^{-1}\right]$ & 3 & $-4 \times 10^{6}\left[\mathrm{~m}^{-2}\right]$ & 0.4 & $-2.3 \pi$ & 0.5 & $-6 \pi$ \\
\hline
\end{tabular}

power, this can be observed in the values of $m$ obtained for the fit.

It was established in [15] that a value of $m=2$ the interaction is considered as local. The physical mechanism that creates such response can be of different origins: heat [20], charge carriers [21], atoms [22]. At the power of $1 \mathrm{~mW}(m=2.5)$, implies that the nonlinear phase change is narrower than the intensity distribution, whereas $10 \mathrm{~mW}$ a $m=0.4$ is obtained; therefore, the nonlinear phase change is extended beyond the incident intensity distribution, which means that the change in the nonlinear refraction index takes place in a bigger area than the illumination area. A thermal process occurred, verified by the FLD model (Table 1), by exposing of the photographic film to higher level of intensity.

The results with $1 \mathrm{~mW}$ of incident power were fitted with the same nonlinearity or nonlocality during all the $\mathrm{z}$-scan. However, when the incident power was increased the nonlinear response of the sample changed during the z-scan. In the case of the FLD model, the experimental results for $5 \mathrm{~mW}$ are better reproduced, for negative $\mathrm{z}$, when the sample exhibited the same nonlinearity $(s=4)$ than that of the $1 \mathrm{~mW}$ curve. However, for positive $\mathrm{z}$ the experimental behavior is better reproduced when $s=3$. When $10 \mathrm{~mW}$ were used, the experimental results, for negative $\mathrm{z}$, are better reproduced with $s=2$ and for positive $\mathrm{z}$ with $s=3$. In the case of the NL model, the experimental results for $5 \mathrm{~mW}$ were better reproduced, for negative $\mathrm{z}$, when $m=2.8$, value slightly larger than that for the $1 \mathrm{~mW}$ curve, and $\Delta \Phi_{0}$ of $-6 \pi$ rads. (20 times the value for $1 \mathrm{~mW}$ ). For positive $z$, the experimental results were better reproduced when $m=0.4$ and $\Delta \Phi_{0}$ of $-3 \pi$ radians. The experimental results for $10 \mathrm{~mW}$ were better reproduced, for negative $z$, when $m=0.4$ and $\Delta \Phi_{0}$ of $-2.3 \pi$ radians, and for positive $z$ when $m=0.5$ and $\Delta \Phi_{0}$ of $-6 \pi$ radians.

\section{Conclusions}

Experimental z-scan curves of bleached photographic film were fitted with different theoretical models:

Focal Length Dependent (FLD) and nonlocal (NL). The results demonstrate that it is not possible to fit the experimental results considering only one contribution during the z-scan. Two contributions were needed to reproduce completely the experimental z-scan curves in both models. Each contribution was considered to act mainly in one part of the z-scan through a Heaviside Step function. Good agreement between the experimental and simulated results was obtained.

\section{Abbreviations}

FLD: The focal length dependent model; NL: The nonlocal model; HS: Heaviside step function; pk: Peak; vy: Valley; HSp: Heaviside step function to weight the peaks; $\mathrm{HS}_{v}$ : Heaviside step function to weight the valleys

\section{Acknowledgements}

Authors thank to the Programa para el Desarrollo Profesional Docente, para el Tipo Superior; Red temática: "Optoelectrónica y óptica no lineal" by the support given.

\section{Authors' contributions}

ERL. Author of Focal Length Dependent (FLD) model and writer of the manuscript. $\mathrm{ABO}$. Author of Nonlocal (NL) model and writer of the manuscript. YEBG. Author of the numerical simulations and, also proposed use a Heaviside Step function; reviewer. JADP. Discussion of the physical phenomena, second author of the numerical simulations and reviewer. MLAC. Discussion of the physical phenomena, author of Nonlocal (NL) model and reviewer. MDI. Author of Focal Length (FLD) model, writer and style editor. All authors read and approved the final manuscript.

\section{Competing interests}

The authors declare that they have no competing interests.

\section{Author details}

${ }^{1}$ Benemérita Universidad Autónoma de Puebla, Av. San Claudio y 18 sur, 72570 Puebla, Puebla, Mexico. ${ }^{2}$ Universidad Nacional Autónoma de México, Apdo, Postal 20-364, 01000 Cd. de México, Mexico. ${ }^{3}$ Instituto Nacional de Astrofísica, Óptica y Electrónica, Luis Enrique Erro \# 1, 72840 Tonantzintla, Puebla, Mexico.

Received: 24 May 2016 Accepted: 8 September 2016

Published online: 20 September 2016

\section{References}

1. Wayne, R.: Light and video microscopy, 2nd edn. Academic, San Diego (2014). ISBN 9780124114845

2. Kumar, S., Udupa, D.V., Debnath, A., Prasad, N., Tokas, R.B., Sahoo, N.K.: Influence of surface relief depth on diffraction efficiency of phase gratings recorded in Slavich PFG-01. Optics \& Laser Technology 47, 305-309 (2013)

3. Woo Lim, C., Bae Park, Y., Ho Sohn, S.: Effects of developing agents and activator on the diffraction efficiencies in a reversal bleached transmission hologram derived from Slavich PFG-01. Optics \& Laser Technology. 41(2), 181-185 (2009)

4. Bányász, l.: Higher-order harmonics in bleached silver halide holograms. Opt. Lasers Eng. 44(9), 926-942 (2006)

5. Bányász, I.: Comparison of the effects of two bleaching agents on the recording of phase holograms in silver halide emulsions. Opt. Commun. 267(2), 356-361 (2006)

6. Bányász, I.: Refractive index modulation vs. before-bleach optical density modulation characteristics of silver halide phase holograms. Opt. Commun. 244(1-6), 79-91 (2005)

7. Bányász, I:: On the semi-physical developing effect of the AAC developer on holograms recorded in Agfa-Gevaert 8E75HD emulsions. Opt. Commun. 192(1-2), 27-35 (2001) 
8. Neipp, C., Beléndez, A., Pascual, I.: The influence of the development in silver halide sensitized gelatin holograms derived from PFG-01 plates. Opt. Commun. 173(1-6), 161-167 (2000)

9. Fimia, A., Blaya, S., Carretero, L., Madrigal, R.F., Mallavia, R.: Real time study of development process in holographic emulsions. Opt. Commun. 173(1-6), 195-201 (2000)

10. Reynoso Lara, E., Iturbe Castillo, M.D., Martí-Panameño, E.: Nonlinear optical behavior of developed and bleached photographic film characterized by z-scan technique. Internet Electron J. Nanoc. Moletrón. 5(1), 903-911 (2007)

11. Sheik-bahae, M., Said, A.A., Van Stryland, E.W.: High-sensitivity, single-beam $n_{2}$ measurements. Opt. Lett. 14(17), 955-957 (1989)

12. Sheik-bahae, M., Said, A.A., Wei, T.H., Hagan, D.J., Van Stryland, E.W.: Sensitive measurement of optical nonlinearities using a single beam. IEEE J Quantum Electronics 26(4), 760-769 (1990)

13. Pálfalvi, L., Tóth, B.C., Almási, G., Fülöp, J.A., Hebling, J.: A general Z-scan theory. Appl. Phys. B. 97, 679-685 (2009)

14. Reynoso Lara, E., Navarrete Meza, Z., Iturbe Castillo, M.D., Treviño-Palacios, C. G., Martí-Panameño, E., Arroyo Carrasco, M.L.: Influence of the Photoinduced Focal Length of a Thin Nonlinear Material in the Z-scan technique. Opt. Express 15(5), 2517-2529 (2007)

15. García Ramírez, E.V., Arroyo Carrasco, M.L., Mendez Otero, M.M., Reynoso Lara, E., Chavez-Cerda, S., Iturbe Castillo, M.D.: Z-scan and spatial self-phase modulation of a Gaussian beam in a thin nonlocal nonlinear media. J. Opt. 13, 085203 (2011) (10 pp)

16. Fang, H.L., Swofford, R.L.: The thermal lens in absorption spectroscopy. In: Kliger, D.S. (ed.) Ultrasensitive laser spectroscopy, pp. 175-232. Academic, New York (1983)

17. Pálfalvi, L., Hebling, J.: Z-scan study of the thermo-optical effect. Appl. Phys. B. 78, 775-780 (2004)

18. Balbuena Ortega, A., Arroyo Carrasco, M.L., Méndez Otero, M.M., Reynoso Lara, E., García Ramírez, E.V., Iturbe Castillo, M.D.: Analytical expressions for $z$-scan with arbitrary phase change in thin nonlocal nonlinear media. Opt. Express 22(23), 27932-27941 (2014)

19. Rodríguez Rosales, A.A., Ortega Martínez, R., Arroyo Carrasco, M.L., Reynoso Lara, E., Treviño Palacios, C.G., Baldovino-Pantaleon, O., Ramos García, R., Iturbe Castillo, M.D.: Neither Kerr nor termal nonlinear response of dye liquid cristal characterized by z-scan technique. Mol. Crys. Liq. Crys. 489, 9-21 (2008)

20. Dabby, F.W., Whinnery, J.R.: Thermal self-focusing of laser beams in lead glasses. Appl. Phys. Lett. 13(8), 284-286 (1968)

21. Segev, M., Crosignani, B., Yariv, A., Fischer, B.: Spatial solitons in photorefractive media. Phys. Rev. Lett. 68(7), 923-926 (1992)

22. Suter, D., Blasberg, T:: Stabilization of transverse solitary waves bya nonlocal response of the nonlinear medium. Phys. Rev. A 48(6), 4583-4587 (1993)

\section{Submit your manuscript to a SpringerOpen ${ }^{\circ}$ journal and benefit from:}

- Convenient online submission

- Rigorous peer review

- Immediate publication on acceptance

- Open access: articles freely available online

- High visibility within the field

- Retaining the copyright to your article 